\title{
Classique ou francophone? De la notion de classique appliquée aux œuvres francophones, éds. Corinne Blanchaud
}

\section{Alexandre Calvanese}

\section{(2) OpenEdition}

Journals

Édition électronique

URL : http://journals.openedition.org/studifrancesi/4491

DOI : 10.4000/studifrancesi.4491

ISSN : 2421-5856

Éditeur

Rosenberg \& Sellier

\section{Édition imprimée}

Date de publication : 1 septembre 2016

Pagination : $371-372$

ISSN : 0039-2944

\section{Référence électronique}

Alexandre Calvanese, "Classique ou francophone? De la notion de classique appliquée aux œuvres francophones, éds. Corinne Blanchaud », Studi Francesi [En ligne], 179 (LX | II) | 2016, mis en ligne le 01 septembre 2016, consulté le 18 septembre 2020. URL : http://journals.openedition.org/studifrancesi/ 4491 ; DOI : https://doi.org/10.4000/studifrancesi.4491

Ce document a été généré automatiquement le 18 septembre 2020.

\section{cc) $(9)$}

Studi Francesi è distribuita con Licenza Creative Commons Attribuzione - Non commerciale - Non opere derivate 4.0 Internazionale. 


\title{
Classique ou francophone? De la notion de classique appliquée aux œuvres francophones, éds. Corinne Blanchaud
}

\author{
Alexandre Calvanese
}

\section{RÉFÉRENCE}

Classique ou francophone? De la notion de classique appliquée aux ceuvres francophones, sous la direction de Corinne BLANCHAUD, Amiens, Encrage édition, 2015, «CRTF», 141 pp.

1 Qu'est-ce qu'un «classique» de la littérature? Depuis quand cette notion s'est-elle affirmée? Faut-il la considérer comme absolue-dépendant ainsi de la valeur intrinsèque de l'œuvre - ou plutôt comme relative - c'est-à-dire en rapport avec les conditions de réception, de reconnaissance et de transmission de l'œuvre, qui sont multiples et variables? Peut-on envisager un emploi du terme qui ne soit pas forcément encadré dans une dynamique politico-culturelle bien définie, c'est-à-dire le classique comme expression d'un patrimoine littéraire et linguistique national? De quelle manière peut-il s'adapter à un contexte si hétérogène et avec une histoire relativement récente comme celui de la francophonie? Voici quelques-unes des questions qui animent les réflexions contenues dans cette courte mais intéressante publication dirigée par Corinne BLANCHAUD, qui rassemble des contributions d'universitaires issus d'espaces différents du monde francophone.

2 Dans sa préface (pp. 7-35), qui définit les enjeux du débat tout en dialoguant avec les autres contributions, Blanchaud rappelle que le terme "classique" n'a pas toujours identifié le même objet: au fil des siècles il a pu avoir une acception sociologique ("classique" a d'abord indiqué l'écrivain de première classe - le classicus scriptor - et ensuite l'œuvre enseignée en classe), une valeur normative (classique, dans la 
définition de Gide, est l'écrivain qui se distingue par la concision, l'ordre, la mesure, la simplicité de son écriture) ou au contraire il a pu s'appliquer à des œuvres capables de porter un défi à la tradition (comme la Recherche de Proust).

Violaine HOUDART-MEROT (Métamorphoses du patrimoine littéraire en France: du classicus scriptor au classique francophone, pp. 37-54) rappelle à son tour que le mot a pris au cours du Grand Siècle la connotation de modèle digne d'imitation, pour devenir au début du $\mathrm{xIX}^{\mathrm{e}}$ synonyme d'art désuet, dépassé: c'est Stendhal qui utilise "classicisme" en opposition à "romantisme", au moment même où l'idée s'impose d'un patrimoine culturel national capable de rivaliser avec les Anciens. Avec Sainte-Beuve, l'idée assume un caractère universel (un classique est un auteur qui «enrichit l'esprit humain»); Gide, au début $\mathrm{du} \mathrm{xx}^{e}$ siècle, récupère l'approche historique et assimile le classique au génie français. Dans des formules comme "classiques africains" ou "classiques francophones", employées par le monde de l'édition pour définir des écrivains incontournables, la notion semble récupérer son sens originel de scriptor classicus. Voici donc que l'association de "classique" et de "francophone" vient briser «l'alliance [...] entre classique, nation et langue française» (p. 50), et demande l'élaboration de "critères de valeur qui ne soient pas exclusivement nationaux", tout comme une remise en question des idéologies qui président à l'élaboration de l'histoire littéraire et du patrimoine transmissible. Un passage, ce dernier, qui n'est pas du tout évident quand on considère le caractère centralisateur et uniformisant de la culture française. C'est pour cette raison que Blanchaud suggère de séparer l'idée de classique de celle d'universalité (qui a trop souvent «servi la visée unificatrice et colonisatrice de la France») pour la rattacher, à l'instar d'Italo Calvino, à une idée de durée, c'est-à-dire à la capacité d'une œuvre de susciter des lectures et des réécritures toujours renouvelées.

4 Si la dimension nationale peut avoir une influence stratégiquement positive pour les littératures francophones mineures, c'est justement dans la mesure où elle favorise une reconnaissance de ces mêmes littératures dans leurs espaces d'émergence respectifs, et peut même «contribuer à une meilleure réception française des patrimoines francophones» (p. 27). Car - voici le problème d'arrière-fond évoqué par Blanchaud - la réception des œuvres francophones (lire: non françaises) doit encore escompter, surtout en milieu scolaire et universitaire, de multiples résistances. Cependant la France n'est pas la seule responsable de cette situation, qui peut avoir deux explications: soit les instances nationales de légitimation sont absentes, soit dans des pays bilingues ou trilingues chaque communauté linguistique se tourne vers le patrimoine littéraire du grand pays monolingue voisin de même langue (comme la communauté francophone de Belgique par rapport à la France) au détriment de son patrimoine "local".

5 Ces deux problèmes (la légitimation et la formation d'un patrimoine national) sont le fil rouge qui parcourt les cinq articles successifs, qui abordent des sujets ponctuels dans différents contextes francophones.

6 Laurent DEMOULIN (L'inaccessible "devenir-classique" d'André Baillon, pp. 55-71) propose une analyse des raisons du semi-échec, ou de la semi-réussite, de l'écrivain belge, dont la promesse de devenir classique ne s'est jamais réalisée. Demoulin propose cinq critères d'accession au titre de classique (entendu dans le sens d'écrivain passé à la postérité) pour tracer le profil de Baillon: l'importance quantitative de l'œuvre; son originalité; sa réception; la situation de l'écrivain au point de vue institutionnel; la biographie légendaire de l'auteur. 
Lucie JOUBERT, dans La place des femmes parmi les "classiques": faut-il en rire ou en pleurer? (pp. 73-83), se penche sur la disproportion éclatante entre la présence masculine et féminine dans les grandes collections éditoriales (voir la «Bibliothèque de la Pléiade»). Joubert plaide-sans grande originalité, comme elle-même le reconnaît-pour la création d'une bibliothèque entièrement composée de classiques féminins comme ultime ressource pour assurer la publicité, la diffusion et la conservation d'un patrimoine-cette fois-ci non national mais «de genre»-autrement condamné à l'oubli.

8 Paul ARON propose une enquête sur L'enseignement des classiques en Belgique francophone (pp. 85-99). L'hypothèse qu'il défend est que l'absence de classiques en Belgique dépend du fait qu'historiquement l'enseignement dans ce pays n'a pas été structuré de manière à produire des auteurs classiques. Deux périodes sont prises en considération par Aron: de 1830 à 1980 et les trois dernières décennies du Xxe, marquées par l'expérimentation de l'enseignement «Rénové».

Roger FRANCILLON (Les avatars de la Chrestomathie Vinet et la difficile constitution d'un patrimoine romand $d u X_{X I}{ }^{e}$ siècle à nos jours, pp. 101-115) montre le curieux phénomène par lequel des écrivains liés à la Suisse française comme Rousseau, Constant et Mme de Staël ont dû attendre la première moitié $d u x^{e}$ siècle pour être considérés comme partie intégrante du patrimoine littéraire romand. Ce-dernier d'ailleurs, dans une Confédération dans laquelle chaque canton jouit d'une extrême liberté dans le choix des programmes, a beaucoup de difficultés à se constituer.

10 Dans le dernier article (pp. 117-127), Christiane CHAULET-ACHOUR présente le projet du Dictionnaire des écrivains francophones classiques paru en 2010 chez Champion, conçu comme un outil à la disposition des enseignants pour approcher des littératures et des auteurs injustement méconnus. Dans ce cas, l'appellatif de "classique francophone" veut signaler aux lecteurs de langue française, aux éditeurs et surtout aux enseignants, des auteurs incontournables qui, de par le monde, ont écrit et créé en français, et qui méritent absolument d'être lus, publiés et enseignés à l'école et dans les cours universitaires (où, actuellement, l'enseignement des littératures francophones «repose sur des bases très fragiles», p. 121).

11 La fortune du "classique francophone", selon Blanchaud, dépendrait ainsi de la capacité du texte de vivre dans la longue durée et de surmonter les obstacles qui empêchent sa reconnaissance immédiate. Marcel Proust, devenu un classique par excellence après avoir été un novateur marginalisé, au début de sa carrière, par le centre de légitimation de l'époque, disait que «tout art véritable est classique, mais les lois de l'esprit permettent rarement qu'il soit, à son apparition, reconnu comme tel». Cependant, les réflexions de cet ouvrage semblent indiquer que les textes francophones non français doivent toujours surmonter quelques obstacles en plus pour accéder à ce statut. 